\title{
DERECHOS DE LA NATURALEZA Y JUSTICIA ECOLÓGICA INTERGENERACIONAL
}

\author{
RIGHTS OF NATURE AND INTERGENERATIONAL ECOLOGICAL JUSTICE
}

\author{
Alicia Morales Lamberti \\ (Universidad Nacional de Córdoba / Argentina) \\ amlamberti327@gmail.com
}

Recibido: $15 / 09 / 2018$

Aprobado: 30/10/2018

\begin{abstract}
RESUMEN
El reconocimiento de la función ambiental de los derechos en el ámbito del paradigma de la justicia ambiental intergeneracional, se ha visto influenciado progresivamente por la coexistencia de otro paradigma no necesariamente excluyente de aquél, basado en el reconocimiento de valores intrínsecos y derechos de la naturaleza, en el marco de una justicia ecológica intergeneracional.

Los derechos intergeneracionales y el reconocimiento de los derechos de la naturaleza, emancipada de su consideración como objeto (bien jurídico) y reconocida como sujeto de derechos, se presentan como derechos de cuarta y quinta generación que cuestionan las prevalentes concepciones antropocéntricas del derecho ambiental.

En ese marco, el análisis se centra en el progresivo reconocimiento normativo y jurisprudencial en numerosos países, de los valores intrínsecos y derechos de la naturaleza en el marco de una justicia ecológica intergeneracional, interpela acerca de los nuevos paradigmas que distinguen a actuales debates jurídicos y políticos, en torno a la relación entre los derechos humanos y el medio ambiente.
\end{abstract}

Palabras claves: función ecológica de los derechos. Naturaleza. Justicia ambiental. Justicia ecológica.

\section{ABSTRACT}

The recognition of the environmental role of rights within the paradigm of intergenerational environmental justice has been progressively influenced by the coexistence of another paradigm not necessarily exclusive to it, based on the recognition of intrinsic values and rights of nature, within the framework of intergenerational ecological justice.

Intergenerational rights and the recognition of the rights of nature, emancipated from its consideration as an object (legal right) and recognized as subject of rights, are presented as rights of fourth and fifth generation, that challenge the prevailing anthropocentric conceptions of environmental law.

In this context, the argument will focus in the progressive legal and jurisprudential recognition in many countries of the intrinsic values and rights of nature in the framework of an intergenerational ecological justice, challenges the new paradigms that distinguish current legal and political debates, about the relationship between human rights and the environment. 
Keywords: ecological function of rights. Nature. Environmental justice. Ecological justice.

\section{Introducción}

Hemos ingresado en un nuevo período geológico que necesita una nueva nominación: Antropoceno (Crutzen- Stoermer, 2000:17), en el que muchas condiciones y procesos vitales se ven profundamente alterados por la actividad humana.

Los últimos avances científicos subrayan la necesidad de tomar en consideración cuidadosamente el efecto agregado a largo plazo de las actividades humanas en el sistema tierra, que conforma la estructura ambiental que posibilita la vida. El sistema tierra se caracteriza por reacciones no lineales e interacciones complejas entre la biósfera viva, compuesta por los ecosistemas y las especies, y los procesos físicos.

El sistema climático no es un mero fenómeno atmosférico. Según el IPCC (2014), la temperatura media de la superficie de la Tierra aumentará entre $1,39^{\circ}$ y $5,78^{\circ}$ Celsius en los próximos 100 años, si no se reducen las emisiones de gases de efecto invernadero. Aunque un aumento de unos pocos grados pueda parecer poco importante, cabe señalar que la temperatura media de la Tierra no ha variado en más de 1 grado en los últimos 10.000 años. Unos pocos grados de variación en la temperatura media determinan la diferencia entre una era glacial y el clima actual de la Tierra.

En la era tecnológica actual, se asiste a un empobrecimiento de la relación histórica entre los seres humanos y la naturaleza. La pérdida de la biodiversidad, la desertificación, el cambio climático y la alteración de ciclos naturales son parte de los costos derivados de nuestro desprecio por la integridad de los ecosistemas y sus procesos vitales.

Ante ello, la Organización de Naciones Unidas, aboga por un cambio. Ante todo, pide que la humanidad trate al planeta con respeto. Ese respeto se materializará únicamente cuando los humanos cambien la manera de percibir su relación con la naturaleza (ONU, 2012).

En ese contexto, el reconocimiento de la función ambiental de los derechos en el ámbito del paradigma de la justicia ambiental intergeneracional, se ha visto influenciado progresivamente por el surgimiento y coexistencia de otro paradigma no necesariamente excluyente de aquél, basado en el reconocimiento de valores intrínsecos y derechos de la naturaleza, en el marco de una justicia ecológica intergeneracional.

Los derechos intergeneracionales y el reconocimiento de los derechos de la naturaleza, emancipada de su consideración como objeto (bien jurídico) y reconocida como sujeto de derechos, se presentan como derechos de cuarta y quinta generación que cuestionan las prevalentes concepciones antropocéntricas del derecho ambiental.

El progresivo reconocimiento normativo y jurisprudencial en numerosos países, de los valores intrínsecos y derechos de la naturaleza, en el marco de una justicia ecológica intergeneracional, interpela acerca de los nuevos paradigmas que distinguen a actuales debates jurídicos y políticos, en torno a la relación entre los derechos humanos y el medio ambiente.

\section{De una ontología dualista a la construcción de ontologías relacionales abiertas: estado de la legislación y jurisprudencia}

El reconocimiento de derechos para la naturaleza, se evidencia en varios países que empezaron a abandonar la visión antropocéntrica de la naturaleza que imperó durante tantos siglos. Un giro ya se vislumbra en el mismo campo del derecho ambiental, a través de la admisión de actuaciones judiciales en beneficio de entes naturales o incluso el acceso de estos mismos a la justicia mediante instituciones 
tutelares, relevantes frente al argumento usual de que no cabe reconocer derechos en favor de entidades sin capacidad para determinarse por sí mismas ni de actuar por tanto con título propio en propia defensa.

Luego del voto de William Douglas en la causa "Sierra Club" (Sierra Club v. Morton, 405 U.S. 727, 1972) y del precedente de Christopher Stone (1972), donde se argumentaba esencialmente a favor del standing de los seres, objetos o zonas naturales afectados por amenazas o daños ecológicos, pero no se excluía la posibilidad y conveniencia de otorgar personalidad jurídica al ambiente o a la naturaleza en general, en diversas normas comunales de los Estados Unidos se viene reconociendo a la naturaleza como sujeto de derechos y específicamente, "el derecho jurídicamente exigible de los ecosistemas naturales a existir y prosperar".

El concepto legal de los derechos de la naturaleza, señala la influencia de la cosmovisión de los pueblos originarios del mundo como actores políticos. Pero en esta construcción de ontologías relacionales abiertas -para distinguirlas de las perspectivas antropocéntricas que corresponderían a una ontología dualista (naturaleza/sociedad)-, no subyacen únicamente cuestiones de reivindicaciones indígenas, sino que expresa inconformidades culturales que cruzan transversalmente muchas culturas y países: con ellas las categorías personas/objetos desaparecen ya que los humanos y los no-humanos pueden ser todos ellos agentes morales, con capacidades análogas, todo integrantes de una misma comunidad expandida, sujetos de derechos y por lo tanto demandantes de justicia (Gudynas, 2010).

El dualismo del antropocentrismo, es suplantado por redes relacionales que integran en igual jerarquía a distintos seres vivos u otros componentes del ambiente (Gudynas, 2010). Se conforman así, comunidades que son tanto sociales como ecológicas, obligando a pensar desde otros puntos de partida cómo se concibe jurídicamente el mundo natural.

Entre esas nuevas concepciones jurídicas, resalta la nueva Constitución del Ecuador (2008) El artículo 71 establece que "la Naturaleza o Pacha Mama, donde se reproduce y realiza la vida, tiene derecho a que se respete integralmente su existencia y el mantenimiento y regeneración de sus ciclos vitales, estructura, funciones y procesos evolutivos", y que toda persona, comunidad, pueblo o nacionalidad podrá exigir a la autoridad pública el cumplimiento de los derechos de la naturaleza. El artículo 72 establece que "la naturaleza tiene derecho a la restauración".

Por su parte, el Estado Plurinacional de Bolivia aprobó una nueva Ley de Derechos de la Madre Tierra (Ley 071, 2010): "Para efectos de la protección y tutela de sus derechos, la Madre Tierra adopta el carácter de sujeto colectivo de interés público. La Madre Tierra y todos sus componentes incluyendo las comunidades humanas son titulares de todos los derechos inherentes reconocidos en esta Ley. La aplicación de los derechos de la Madre Tierra tomará en cuenta las especificidades y particularidades de sus diversos componentes. Los derechos establecidos en la presente Ley, no limitan la existencia de otros derechos de la Madre Tierra" (art. $5^{\circ}$ ).

Inspirada en la legislación anterior, en el Distrito Federal de México en el año 2013 entró en vigor la Ley Ambiental de Protección a la Tierra, para proteger a la Madre Tierra, que posee estatus de un ser vivo: "Art. 86 bis 1. La Tierra es un sistema viviente dinámico conformado por la comunidad indivisible de todos los sistemas de vida y los seres vivos, interrelacionados, interdependientes y complementarios, que comparten un destino común". A los fines jurídicos de su protección y tutela "la Tierra adopta el carácter de ente colectivo sujeto de la protección del interés público. En su aplicación se tomarán en cuenta las especificidades y particularidades de sus diversos componentes" (Art. 86 bis $3)$.

Como puede observarse, esas ideas presentan una ruptura revolucionaria en la comprensión antropocéntrica convencional y un realineamiento de cómo se valora el mundo natural, pero no se trata de una experiencia aislada, por cuanto se viene replicando por pueblos que tienen una comprensión de sí mismos y un punto de partida muy diferentes. 
Así, ese reconocimiento de la naturaleza como sujeto de derechos, reconoce variadas experiencias normativas, donde el entorno natural merece ser protegido en sí y por sí mismo (Suecia, 1999) por cuanto posee valor intrínseco, independiente del valor que su uso pueda tener para el hombre (Noruega, 2010).

A diferencia de Ecuador, los derechos de la naturaleza en Nueva Zelanda no están reconocidos a nivel constitucional, las comunidades nativas fueron fundamentales en la creación de nuevos marcos legales que reconocen como sujetos de derecho a territorios y ecosistemas, otorgando personalidad jurídica al Parque Te Urewara. Esto fue parte de los esfuerzos de reparación por la injusticia histórica generada cuando el Estado de Nueva Zelanda fue fundado: la conquista colonial de las tierras de los pueblos originarios. La Ley de Te Urewara (2014) reconoce al Parque como sujeto de derechos, lo declara hogar sagrado del pueblo Tuhoe y parte integral de su cultura e identidad, al mismo tiempo que es de valor intrínseco para todos los neozelandeses.

En un proceso judicial emancipador similar, una tribu maorí local ayudó al río Whanganui a obtener el estatus de persona jurídica en 2014 y se reconoció el derecho a su gestión como su guardián legítimo. Con ello, se reconoció entre otras cosas, que el río Whanganui era un ser vivo y que las personas y el río eran inseparables, y se le concedió reconocimiento legal como "entidad jurídica con capacidad procesal por derecho propio".

En relación a Ecuador, el primer caso en que se tuvo conocimiento de aplicación judicial de los derechos de la naturaleza se dio en el año 2011. La Acción de Protección, fue interpuesta en contra del Gobierno Provincial de Loja "a favor de la Naturaleza, particularmente a favor de la cuenca del río Vilcabamba". El gobierno local había iniciado un proyecto de ampliación de carreteras y vertía restos en el río, lo que afectaba a su flujo y causaba contaminación. El tribunal decidió que se habían violado los derechos constitucionales del río a fluir y obligó al gobierno provincial a reparar los daños.

En igual sentido, se han conferido diversas medidas cautelares a favor de los Derechos de la Naturaleza, debido a la fragilidad de los ecosistemas existentes en Galápagos. En un caso, se tuvo en cuenta el régimen especial que rige en esta zona insular respecto a la limitación de actividades que puedan afectar el medio ambiente, sin contar con licencia ambiental y social (Estero Wincheles en Esmeraldas). Asimismo, se hizo lugar a una Acción de Protección por desarrollo de actividades mineras sin licencia ambiental, que provocaban el deslizamiento de materiales en el Río Granobles (Río Blanco), causando su afectación.

Más recientemente (2016) la Corte Constitucional de Colombia, reconoció al río Atrato, su cuenca y afluentes como una "entidad sujeto de derechos a la protección, conservación, mantenimiento y restauración a cargo del Estado y las comunidades étnicas" en calidad de sus guardianes.

Un precedente notable fue sentado por el Tribunal Supremo de la provincia india de Uttarakhand. En su sentencia recaída en la causa T. N. Thirumulpad Godavarman v. Union of India (2017), consideró que para hacer efectiva la justicia ambiental era necesario aplicar principios ecocéntricos en vez de antropocéntricos. La Corte determinó que "la cordillera del Himalaya, sus glaciares, ríos (Ganges y Yamuna principalmente), caídas, corrientes, lagos, junglas, bosques, praderas, valles, humedales y manantiales habrían de ser considerados como personas jurídicas a fin de garantizar su sobrevivencia, seguridad, sustentabilidad y resurgimiento" y estableció expresamente que "los derechos de estas últimas debían considerarse equivalentes a los de los seres humanos y repararse de igual manera".

Por otra parte, el reconocimiento de los derechos de los animales, se ha desarrollado en forma paralela al proceso de afirmación de los derechos de la naturaleza. Valga como ejemplo, el caso de la orangutana Sandra, quien siendo reconocida como "ser sintiente" (novedosa categorización introducida por la reforma de 2015 del Código Civil francés), "es una 'persona no humana', y por 
ende, sujeto de derechos y consecuentes obligaciones hacia ella por parte de las personas humanas" (Morales Lamberti, 2017) ${ }^{2}$.

\section{Función ecológica de los derechos y derechos de la naturaleza: el hilo de Ariadna y las necesidades jurídicas insatisfechas}

El derrotero histórico de la relación hombre-ambiente estuvo constantemente marcado por un sustrato cultural o código ético adaptado a la medida del hombre: es él quien fue dictando las reglas de un antropocentrismo fuerte legitimador de cada forma de dominación.

La posibilidad de una regulación jurídica que atienda al mundo de la naturaleza, que consista en separar el todo en partes y luego realizar un ejercicio de recomposición, reuniendo todos esos factores en una sola formulación normativa, no fue fácil de construir y menos fácil de comprender. Implicaba nuevas instituciones, nuevos criterios de justicia, nuevas leyes y especialmente nuevos métodos: era necesario cambiar el microscopio jurídico sustancial y procesal pensado para la resolución de conflictos individuales patrimoniales, por el "macroscopio", apto para resolver las cuestiones complejas y holísticas que plantean los retos ambientales. Acudir al "macroscopio" entonces, es, antes que nada, un sistema de pensar y, después que nada, un sistema de pensar la naturaleza.

No escapa a este reconocimiento, que los conflictos ambientales emergen de intereses y estrategias diferenciadas de relacionamiento, apropiación y aprovechamiento de la naturaleza y por ende, de políticas públicas, que avecinan una fuerte crisis de instituciones y de sistemas jurídicos tradicionales basados en una racionalidad retórica e instrumental. Tampoco desconoce, que la lista de indicadores ambientales que cuestionaban y cuestionan la sustentabilidad de la vinculación del hombre con la naturaleza es muy vasta y que esto es un hecho antrópico y no ecológico, al cual el derecho ambiental también debe dar respuestas, porque estamos viviendo por sobre los límites de nuestra tarjeta de crédito ecológico (Morales Lamberti, 2017).

\section{El derecho a tener derechos}

La abolición de la esclavitud de la naturaleza ha sido comparada con la abolición de la esclavitud humana (Stutzin, 1984:98). Teólogos y juristas mantuvieron durante siglos la tesis que los indígenas americanos no eran personas y carecían de alma. Aun en el año 1858 un tribunal de Virginia (Estados Unidos), declaró que "un esclavo no es una persona, sino una cosa".

Así como las leyes esclavistas, que convirtieron a seres humanos en propiedad afianzaron una relación de explotación, nuestros sistemas jurídicos han mantenido en sucesivos períodos históricos, sectores relegados de la sociedad y de sus derechos, como los pueblos originarios, los negros, los gays, las mujeres... y con ellos también a la naturaleza, que también es mujer y es mujer sojuzgada.

Pero más que multiplicar ejemplos históricos o actuales, justo es reconocer que todo ello se basa en la construcción de realidades jurídicas a partir de supuestos culturales, que los juristas llamamos ficciones jurídicas. A lo largo de la historia y aún en la actualidad, la sociedad construye categorías y otorga características a todo lo que la circunda: Los modos en que categorizamos tienen su origen en la sociedad misma, y los modos de ver la realidad y de actuar sobre ella están permeados por los modos en que clasificamos esta realidad.

La construcción y la asimilación de las ficciones jurídicas son contextuales. Por ello, cada decisión legislativa o judicial en materia ambiental, es parte de distintas percepciones y diversas construcciones de la sociedad y también, del pluralismo en la construcción social de la realidad. La vieja formulación del derecho positivo de considerar sólo a los seres humanos (personas) como únicos sujetos de

\footnotetext{
${ }^{2}$ El término surge de Joël de Rosnay en "El Macroscopio", A.C., Madrid, 1977.
} 
relaciones jurídicas (posteriormente ampliada a la "ficción" de las personas jurídicas y a la subjetivación jurídica de "patrimonios de afectación"), continúa formulando resistencias al reconocimiento de los derechos de la naturaleza y su condición de sujeto de derechos.

Es que los modos categorizar y clasificar encierran relaciones de poder específicas, que a su vez pueden provocar relaciones de desigualdad, dominación y sometimiento de seres vivientes, que aún hoy deja por fuera a los indígenas, los físicamente discapacitados, la naturaleza y las generaciones futuras, entre otros.

Eso interpela ciertos modos de ver y actuar sobre la vida de los otros humanos y no humanos. Por ello, la lucha por los derechos de la naturaleza y su condición de sujeto de derechos, no es sólo un recurso técnico cuya función jurídica radica en la posibilidad de lograr soluciones a problemas nuevos mediante la utilización de construcciones creadas para enfrentar situaciones distintas, sino también una manera de hacer visible a uno entre otros tantos excluidos.

\section{El paradigma ecológico}

La modernidad transformó la naturaleza en "medio ambiente", un "entorno" objeto externo al ser humano, haciendo del hombre el centro del mismo, su dueño. Con ello perdimos el sentido del vínculo y del límite en las relaciones con la naturaleza. Vínculos como alianzas, ataduras, ligazones, anclaje y enraizamiento. Límite como lindero, umbral que no se cruza, valor límite de donde emergen dos grandes dimensiones que revelan esta relación: la que hace de la naturaleza un objeto y la que la transforma en sujeto.

Las últimas teorías científicas también ofrecen un nuevo entendimiento de la relación entre los seres humanos y su entorno. El supuesto de que nuestra especie es distinta e inherentemente superior a otras formas de vida o de que tenemos un lugar y una función privilegiados en el cosmos no tiene ninguna base científica (ONU, 2012).

En el abordaje actual, el paradigma ambiental completa la teoría de los derechos. Esta nueva "ontología" da lugar al surgimiento de deberes de protección de los bienes colectivos y a límites en el ejercicio de los derechos individuales que surgen cuando afectan al bien colectivo (ambiente) de modo irreversible (Lorenzetti, 2008). Es este el estadio de la mayoría de las leyes ambientales, que en última instancia, no hacen más que regular la velocidad a la cual la destrucción del medio ambiente puede llevarse a cabo.

Con ello, buena parte de los derechos humanos resultan incompletos, porque no logran establecer un vínculo entre la parte (el individuo) y el todo: la sociedad y la biósfera. Consecuentemente, la justicia que genera es una que se desenvuelve exclusivamente entre humanos, donde el bien ambientenaturaleza es un conjunto de objetos. Se defiende la calidad de vida de o el ambiente en función de las personas, alejados de los valores intrínsecos del ambiente ya que es parte de la perspectiva antropocéntrica.

Esas limitaciones, no han estado ajenas al Derecho Ambiental, que, como disciplina portadora del paradigma ecológico, ha encontrado resistencias en el mismo sistema jurídico prevalente, las cuales se tradujeron durante décadas, en su marginalidad académica, judicial y doctrinaria. Como heredero de dispersas disposiciones sobre el uso y explotación de los recursos naturales, sus piezas legislativas permanecieron ajenas al reconocimiento y significación de la naturaleza como sujeto de derechos.

A nivel internacional, la seguridad ecológica, no surgió sino hasta la Conferencia de Estocolmo sobre el Entorno Humano en 1972, pero signada por una visión antropocéntrica, basada en el paradigma del excepcionalismo humano, que considera que el ser humano no forma parte del medio ambiente y es el dueño del planeta. 
La Organización de las Naciones Unidas, tuvo la posibilidad de un cambio de paradigma en 1982. La iniciativa de la Estrategia Mundial para la Conservación culminó con la aprobación de la Carta Mundial de la Naturaleza. La Carta afirma que "la especie humana es parte de la naturaleza y que la vida depende del funcionamiento ininterrumpido de los sistemas naturales" (Res. AG 37/7). Desde entonces, se reconoce que la humanidad es una de las especies constitutivas de la naturaleza y que su propia existencia guarda dependencia de su simbiosis con ella, así como que toda forma de vida tiene un valor intrínseco y merece, por tanto -cualquiera que sea su utilidad-, el debido respeto por parte de la especie humana. Con ello, se incluyen los derechos de la Tierra y de todos los seres a "la vida y a existir" y a "la identidad y salud integral".

Más recientemente, una contribución a la visión biocéntrica moderada, relativa a la armonía con la naturaleza en la promoción del desarrollo sustentable, fue el reconocimiento en el documento final de la Conferencia de las Naciones Unidas sobre el Desarrollo Sostenible, de la relación entre la humanidad y la tierra, reafirmando que "la existencia humana es parte inextricable de la naturaleza".

Con la inclusión del ambiente en los derechos humanos de tercera generación, emerge el paradigma ambiental, que se basa en una idea de interacción compleja y que toma en cuenta los efectos individuales y colectivos, presentes y futuros de la acción humana (derechos de cuarta generación o generaciones futuras), que representa para los individuos, un sistema donde predominan los deberes y los límites a los derechos en razón de la protección que demanda el bien colectivo.

Progresivamente el biocentrismo, fue incorporando la mirada ecocéntrica y los valores intrínsecos, cuestionando los supuestos epistemológicos y jurídicos tradicionales que reducen la naturaleza a sus características físicas observables que posee un valor de cambio, como medida de todas las cosas.

Si la naturaleza posee valores objetivos, independientes de las valoraciones y prestación de utilidades para los seres humanos, al elenco de los conflictos ambientales de tipo distributivo y sus correlativos principios y reglas jurídicas basados en la equidad distributiva, se adicionan conflictos ontológicos que requieren ponderar la relación de orden intersubjetivo de los humanos con los elementos naturales no humanos.

Como se observa, los derechos de la naturaleza, así como los derechos humanos, derivan de la misma fuente de existencia, y resultan compatibles entre sí. Estos derechos no se oponen a los derechos humanos: como parte de la naturaleza, nuestros derechos se derivan de esos mismos derechos. El derecho humano a la vida carece de sentido si los ecosistemas que nos mantienen carecen del derecho a existir. No hay derechos humanos si no se cuenta con un hábitat donde el hombre pueda vivir con dignidad: esencia y núcleo duro de los derechos humanos.

Esa perspectiva ecocéntrica de los derechos propios de la naturaleza no invalida, sino que acompaña y refuerza, la perspectiva biocéntrica clásica de los derechos humanos que se extienden sobre el ambiente. Estos incluyen, por ejemplo, el derecho a un ambiente sano y ecológicamente equilibrado (Gudynas, 2010:52).

La inclusión de los derechos de la naturaleza en nuestros sistemas jurídicos, mediante el reconocimiento de los derechos fundamentales de los ecosistemas y las especies a existir, prosperar y regenerarse, implicaría que los sistemas jurídicos promuevan un balance ecológico vital, al equilibrar los derechos humanos frente a los derechos de los demás miembros de la comunidad biótica.

El reconocimiento de los derechos de la naturaleza, a que se respete integralmente su existencia y el mantenimiento y regeneración de sus ciclos vitales, estructura, funciones y procesos evolutivos -leído en clave política-, por una parte afirma una valencia emancipatoria de la naturaleza, según diversas cosmovisiones (dimensión epistemológica), pero también evidencia diferentes percepciones sobre una misma realidad (dimensión ontológica). 
En definitiva, la objeción contra el reconocimiento de la titularidad de derechos de la naturaleza en base diversas posiciones discursivas no es más que la reiteración de la primaria reacción que a lo largo de la historia se ha registrado ante cualquier noticia que le advierte al humano que no es tan centro ni tan privilegiado como se ha creído: desde Copérnico, pasando por Galileo Galilei, hasta Darwin o Freud viene pasando lo mismo. El narcisismo humano tiende a radicalizar las posiciones supuestamente defensoras del humanismo hacia un antropocentrismo que raya en los límites del exabrupto cartesiano (Stutzin, 1984).

\section{Justicia ambiental y justicia ecológica}

Si bien la distinción entre dos justicias -una ambiental y otra ecológica- es reciente, las múltiples disposiciones normativas que existen y el enfoque pluralista que promueve en nuestro caso, la propia Constitución Nacional, hacen que su relación (formal) sea dinámica y en permanente evolución, permitiendo la coexistencia del paradigma ambiental con el paradigma ecológico, basado en el reconocimiento de valores intrínsecos y derechos de la naturaleza, en el marco de una justicia ecológica intergeneracional.

Es posible identificar al menos tres aproximaciones teóricas que enraízan en el derecho ambiental argentino: En primer lugar el enfoque antropocéntrico (derecho a un ambiente sano, equilibrado, apto para el desarrollo humano art. $41 \mathrm{CN}$ ) y en segundo lugar, una visión biocéntrica intergeneracional, que reivindica concepciones más globales y solidarias de la responsabilidad humana, que abogan -en igual medida- por los deberes del hombre con la naturaleza y las generaciones venideras: "las actividades productivas deben satisfacer las necesidades presentes sin comprometer las de las generaciones futuras".

Pero también, se distinguen precisos enfoques ecocéntricos, que si bien conciben a la naturaleza como bien colectivo (objeto), su protección y restauración se fundamenta en valores intrínsecos propios de un sujeto de derechos, que permiten cosmovisiones plurales y alternativas a los planteamientos anteriores (el daño ambiental generará la obligación prioritaria de recomponer (art. $41 \mathrm{CN}$ ). En este sentido, la compensación ecosistémica comporta un tipo de restitución aplicada exclusivamente a la naturaleza.

Esta visión ecocéntrica, a modo de ejemplo, claramente se recepta en varias normas de la Ley General del Ambiente 25,675), entre cuyos objetivos está "mantener el equilibrio y dinámica de los sistemas ecológicos"; "posibilitar la sustentabilidad ecológica"(art. $\left.1^{\circ}\right)$; “(...) garantizar la dinámica de los sistemas ecológicos, mantener su capacidad de carga" (art. $6^{\circ}$ ); y la concepción de daño ambiental colectivo: "Se define el daño ambiental como toda alteración relevante que modifique negativamente el ambiente, sus recursos, el equilibrio de los ecosistemas, o los bienes o valores colectivos".

Más ampliamente, el art. 240 del nuevo Código Civil y Comercial, condensa una clara concepción ecocéntrica cuando establece que el ejercicio de los derechos individuales no sólo debe ser compatible con los derechos de incidencia colectiva, sino especialmente "no debe afectar el funcionamiento ni la sustentabilidad de los ecosistemas de la flora, la fauna, la biodiversidad, el agua, los valores culturales, el paisaje, entre otros, según los criterios previstos en la ley especial".

Dicho en otras palabras: el medio ambiente, las generaciones futuras y la naturaleza son un elemento transversal al derecho ambiental argentino.

Mientras la justicia ambiental se basa en los derechos a un ambiente sano o la calidad de vida, reconociendo las categorías jurídicas de bienes y derechos colectivos relativos al ambiente desde una perspectiva sujetos-objeto, y está destinada a resolver conflictos en una dimensión distributiva de desigual acceso social a recursos, bienes y servicios ambientales; la justicia ecológica parte de reconocer a la naturaleza desde sus valores propios: un objeto que quiere ser sujeto y generar relaciones intersubjetivas (sujeto-sujeto), que posee valores intrínsecos y sus propios derechos a que se 
respete integralmente su existencia, mantenimiento y regeneración de sus ciclos vitales, estructura, funciones y procesos evolutivos.

Si bien la recepción de esta perspectiva en los debates jurídicos es limitada, ya que prevalecen la perspectiva de la justicia ambiental, ésta no ofrece verdaderas soluciones en un contexto inter y multicultural donde otros saberes definen su comunidad de agentes morales y políticos de manera más amplia, integrando a los no-humanos, tal como ocurre con nuestros pueblos originarios.

La Madre Tierra, como una comunidad indivisible vital de seres interdependientes e interrelacionados con un destino común. La Madre Tierra es un ser vivo. La Madre Tierra es una comunidad única, indivisible y autorregulada, de seres interrelacionados que sostiene, contiene y reproduce a todos los seres que la componen, en la que cada ser se define por sus relaciones como su parte integrante.

Así como los seres humanos son titulares de derechos humanos, todos los demás seres de la Madre Tierra también tienen derechos que son específicos a su condición y apropiados para su rol y función dentro de las comunidades en los cuales existen. En el plano procesal, es evidente que la representación de los derechos de la naturaleza no será ejercida por olivos o delfines, sino por individuos que actúan en representación de éstos, en razón que acceso a la justicia no es el acceso al tribunal sino a los derechos.

Cuando el derecho positivo emplea el verbo "reconocer" los derechos de la naturaleza, alude a realidades ya existentes, no creadas, sino sólo declaradas por el sistema jurídico, el cual las pone de manifiesto y/o las registra, a fin de formalizar los efectos jurídicos que produce su existencia.

Como todos sabemos, el reconocimiento de derechos no proviene de un acto de conocimiento, no depende de la lógica o de la teoría jurídica, sino de la política y de los equilibrios de poder. La lucha por los derechos de la naturaleza es por eso, no sólo una lucha jurídica, sino política.

\section{Justicia ecológica intergeneracional}

Una aproximación a reglas de justicia entre los seres humanos de diferentes generaciones, puede ser entendido bajo el principio del igualitarismo diacrónico (Pontara, 1996:106), por el que los intereses de los humanos de la generación presente no cuentan más que los de los humanos de generaciones futuras $\mathrm{y}$, en consecuencia, los intereses de todos ellos deberán ser tenidos en cuenta con independencia del momento temporal en que vivan los portadores de esos intereses.

Como hemos visto, el paradigma de la justicia ambiental, aún transita la cornisa de la división naturaleza-cultura como propiedades de la realidad, con un lenguaje que aún no traduce la estrecha relación intersubjetiva que se establece entre determinado pueblo y su entorno.

Como contrapartida, el paradigma de la justicia ecológica recepta un giro ontológico que se aparta del proceso de ordenación y categorización propio de la cultura dominante. La otredad cultural "corresponde a una forma de vida particular de ser, ver y actuar en el mundo", constituida a partir de la cosmovisión y valores espirituales que emanan de una estrecha relación intersubjetiva de pueblos con sus territorios y recursos naturales, en especial los aspectos colectivos de esa relación.

Si aceptamos que la ética tiene algo que ver con la debilidad de los vulnerables y con las desigualdades de poder, y la naturaleza no escapa a esa doble situación, también las relaciones intergeneracionales son un asunto que interesa extraordinariamente a la ética, pero también al derecho.

Con ello, la asimetría sincrónica de la relación intergeneracional humana se traslada también, a los derechos humanos para la protección de los aspectos no humanos del medio ambiente. La aplicación de las obligaciones de derechos humanos a retos ambientales especialmente apremiantes, como el cambio climático, los conflictos armados y los desplazados por motivos ambientales, corren el riesgo 
de dejar de lado importantes aspectos que no se pueden reducir con facilidad a las necesidades y los intereses humanos (derechos de quinta generación o derechos de la naturaleza).

Es que son las poblaciones humanas las que son interdependientes del mundo natural -y no al contrario- y las que deben asumir las consecuencias de sus acciones y omisiones con la naturaleza. Se trata de entender esta nueva realidad sociopolítica con el objetivo de lograr una transformación, así como ha ocurrido antes con los derechos civiles y políticos (primera generación); los económicos, sociales y culturales (segunda generación), y los ambientales (tercera generación).

Las futuras generaciones (cuarta generación), se presentan vulnerables con respecto a nosotros. Estamos por completo fuera de su poder causal, mientras que ellas se hallan sometidas al nuestro. Para ello, debemos mirar a los pueblos Aymaras, quienes ponen al futuro siempre atrás, y adelante ponen al pasado, porque es el que enseña el camino.

Es hora que la naturaleza sea emancipada de su sola condición de hábitat de la especie humana, para concebirla como comunidad de seres vivientes interdependientes unos de otros, donde la justicia con la naturaleza debe ser aplicada más allá del escenario y de la reducida escala humana, para velar por la supervivencia de esta comunidad de seres vivientes.

\section{Reflexiones finales}

El sentido de los vínculos y límites jurídicos con la naturaleza, como componente ontológico del paradigma ecológico, integran tímidamente nuestro sistema jurídico. Pero aún desconocen a la naturaleza como un orden ontológico y una organización material de la que emerge la vida. Persiste en el derecho ambiental, la prevalencia de la naturaleza cosificada como soporte de recursos naturales, materias primas de un proceso productivo, que en el mejor de los supuestos, presta servicios ambientales y se instituye como condición de sustentabilidad de todo orden económico y social.

Los precedentes normativos y jurisprudenciales mencionados, sólo marcan una tendencia mundial cuyos resultados concretos requieren ser ponderados, pues debaten uno de los postulados básicos del dualismo moderno: la reducción de la naturaleza a sus propiedades físicas observables, sustituyéndolo por modelos ontológicos distintos que bajo el paradigma antropocéntrico, tradicionalmente son descartados por ser "irreales", simbólicos o míticos.

El lenguaje del derecho reconoce así, una cierta pluralidad y autodeterminación ontológica, atribuyendo propiedades a elementos naturales y capacidades de relacionamiento sujeto/sujeto, que reclaman una nueva hermenéutica jurídica.

Se trata de un diálogo difícil, donde esos vínculos y límites jurídicos con la naturaleza se traducen en conceptos jurídicos que adquieren un sentido eminentemente político, que tiene como punto de partida el conocimiento de la alteridad de la naturaleza en tanto que superación de su imagen cosificada. Es que la verdad y las formas jurídicas que le corresponden se desprenden y se construyen dentro de formas de saber y formas de poder (Foucault, 1995).

Se requiere entonces, una verdadera ética de la vida y para la vida, desde donde se asume que el hombre no sólo es titular de derechos humanos, sino igualmente titular de responsabilidades que deben ser asumidas individual y colectivamente. Un sistema de gobernanza, podría decirse, basado en un estado de derecho ecológico fundado en un sentido transformado de democracia, en el que tanto las personas como las comunidades acepten su condición de ciudadanos ecológicos del mundo y su responsabilidad de respetar los complejos engranajes de la biósfera. Este tipo de democracia restablecería los vínculos de las personas con los fundamentos ecológicos que las sustentan (ONU, 2012). 
El diálogo entre el derecho y la ecología conduce a una ecologización del derecho y a la consecuente juridización de la ecología" (Ost, 1996:109), a fin que el sistema jurídico sea permeado por una comprensión profunda de la complejidad y procesualidad de los sistemas ambientales.

La historia de los derechos de la naturaleza, como la lucha por los derechos, es un profeta con la mirada vuelta hacia atrás: por lo que fue, y contra lo que fue, anuncia lo que será.

\section{Bibliografía}

Cormac, C. (2011). ¿Tienen los humanos legitimación para negarle derechos a la naturaleza? En Derechos de la Naturaleza y la Naturaleza de sus Derechos, Serie Justicia y Derechos Humanos, Quito, Ecuador.

Crutzen, P. J. y Stoermer E. F. (2000). The Anthropocene. Global Change Newsletter, 41, 2000.

Foucault, M. (1995). La verdad y las formas jurídicas. Barcelona: Gedisa.

Grupo intergubernamental de expertos sobre el cambio climático (IPCC) (2014). Climate Change 2014: Impacts, Adaptation, and Vulnerability.

Gudynas, E. (2010, julio-diciembre). La senda biocéntrica: valores intrínsecos, derechos de la naturaleza y justicia ecológica (CLAES). Tabula Rasa, 13, 45-71. Bogotá, Colombia.

Lovelock, J. (2006). The Revenge of Gaia: Earth's Climate Crisis and the Fate of Humanity. New York: Basic Books.

Morales Lamberti, A. (2017). Derechos de la Naturaleza y justicia ecológica. En Pastorino, L. (Dir). Ciclo de Curso de Postgrado $4^{\circ}$ Curso: Las Formas de la Naturaleza y sus Formas de Regulación. La Plata: UNLP.

Morales Lamberti, A. (2016). Dimensión social y colectiva de los derechos humanos: relacionalidad e influencias del paradigma ambiental. En Cafferatta, N. (Dir). La Dimensión Social del Derecho Ambiental (pp. 407-426). Santa Fe: Rubinzal Culzoni.

ONU (2012, 17 agosto). Informe del Secretario General: Armonía con la Naturaleza. A/67/317.

Ost, F. (1996). Naturaleza y Derecho. Para un debate ecológico en profundidad. Bilbao: Mensajero.

PONTARA, G. (1996). Ética y generaciones futuras. Barcelona: Ariel.

Stone, C. D. ([1972] 2010). ¿Should Trees Have Standing? Law, Morality and the Environment. New York: Oxford University Press.

Stutzin, G. (1984). Un imperativo ecológico: reconocer los derechos de la naturaleza. Ambiente y Desarrollo, I (1), 97-114. 